\title{
Pattern transfer into silicon using sub-10 nm masks made by electron beam-induced deposition
}

\author{
Marijke Scotuzzi, ${ }^{a}$ Martin J. Kamerbeek, ${ }^{a}$ Andy Goodyear, ${ }^{b}$ Mike Cooke, ${ }^{b}$ and Cornelis W. Hagen ${ }^{\mathrm{a}, *}$ \\ ${ }^{a}$ Delft University of Technology, Department of Imaging Physics, Charged Particle Optics Group, Lorentzweg 1, 2628 CJ, Delft, The Netherlands \\ ${ }^{b}$ Oxford Instruments Plasma Technology, North End, Yatton, Bristol BS49 4AP, United Kingdom
}

\begin{abstract}
To demonstrate the possibility of using electron beam-induced deposition (EBID) masks for sub$10 \mathrm{~nm}$ pattern transfer into silicon, first experiments were carried out by using 20- to 40-nm EBID masks, which were etched by different chemistries. It is experimentally verified that recipes based on hydrogen bromide, chlorine, and boron trichloride can selectively etch silicon when using 20- to 40-nm masks made by EBID. We observed an enhancement of the height ratio, i.e., the ratio of the height of structures before and after etching, up to a factor of 3.5 when using chlorine chemistry. To demonstrate the pattern transfer of sub-10 nm structures, further experiments were carried out using 8- to 20-nm EBID masks in combination with hydrogen bromide, chlorine, and fluorine chemistries. Fluorine chemistry provided the best results in terms of surface smoothness and height ratio. In this case, 7.4-nm lines were successfully transferred into silicon, resulting in 14.3-nm-wide lines with a height ratio of $\sim 5$. . 2015 Society of Photo-Optical Instrumentation Engineers (SPIE) [DOI: 10.1117/1.JMM.14.3.031206]
\end{abstract}

Keywords: electron beam-induced deposition; nano pattern transfer; nanofabrication; reactive ion etching; inductively coupled plasma; nano imprint lithography; nano imprint lithography stamps.

Paper 15046SSP received Apr. 15, 2015; accepted for publication Jun. 16, 2015; published online Jul. 15, 2015.

\section{Introduction}

Single-digit nanometer structures, i.e., structures with dimensions $<10 \mathrm{~nm}$, are not easy to fabricate. Standard lithography techniques, such as resist-based electron beam lithography (EBL), do not routinely provide sub-10 nm resolution. Electron beam-induced deposition (EBID) is a highresolution direct write lithography technique that is capable of writing single nanometer patterns. An electron beam is focused onto a sample in a scanning electron microscope (SEM) and made to react with a precursor gas let into the SEM through a nozzle positioned in proximity of the substrate. The gas molecules are dissociated into a volatile part, which is pumped out of the SEM, and a nonvolatile part, which remains on the sample surface, forming a deposit. Typical precursors are $\mathrm{W}(\mathrm{CO})_{6}$ and trimethyl (methylcyclopentadienyl)-platinum (IV) ( $\mathrm{MeCpPtMe}_{3}$ ), which lead to tungsten and platinum deposits, respectively.

The high spatial resolution of EBID has been demonstrated by van Dorp et al., who patterned dots of $1 \mathrm{~nm}$ diameter in a $200 \mathrm{kV}$ scanning transmission electron microscope, using the $\mathrm{W}(\mathrm{CO})_{6}$ precursor on a 30 -nm-thick $\mathrm{Si}_{3} \mathrm{~N}_{4}$ membrane. ${ }^{1}$ Using an SEM, van Oven et al. patterned 2.9-nm FWHM lines on a silicon bulk substrate using the $\mathrm{MeCpPtMe}_{3}$ precursor. $^{2}$

However, EBL techniques are serial writing techniques and are therefore inherently slow. To enhance the writing speed, multibeam systems can be used. Post et al. ${ }^{3}$ demonstrated that the throughput of EBID can be enhanced by a factor of 196, when using a multibeam SEM that delivers $14 \times 14$ beams to the sample surface, with the same characteristics as a state-of-the-art single beam SEM. ${ }^{4,5}$ However, for large-area manufacturing of single nanometer structures and devices, it would be useful when a fast and low-cost technique, such as nano imprint lithography (NIL), could be available. NIL is a nonconventional lithography technique with which nanostructures are fabricated through mechanical deformation of a soft polymer by pressing a mold (or stamp) in it, at a controlled temperature and pressure. NIL is a massively parallel lithography technique and therefore extremely suitable to pattern large areas. However, a major challenge of this technique is the fabrication of high-resolution stamps. In the case of UV-assisted NIL, also known as step and flesh imprint lithography (SFIL), the resist is cured with UV light; therefore, transparent stamps are required. For thermal NIL, the resist is cured thermally; hence, the candidate material for molds can be silicon because of its hardness and high mechanical strength. ${ }^{6}$ High-resolution NIL stamps can be fabricated by depositing features on top of a substrate. Optical lithography is generally used for micro-scale features and EBL for patterning nano-scale features. These deposits form a mask for a subsequent anisotropic reactive ion etch (RIE) into the underlying substrate, leading to the surface features required for NIL molds. Sub-15 nm nano imprint molds can be fabricated by using EBL with hydrogen silsesquioxane resist by either direct deposition of the features on top of the silicon substrate or by transferring these deposits into the underlying silicon substrate, to increase the aspect ratio of the patterns. ${ }^{7}$

We propose to use EBID to fabricate stamps for sub$10 \mathrm{~nm}$ NIL. For SFIL, direct deposition of a UV transparent material on a transparent substrate would be possible, but it requires a suitable precursor that leads to transparent deposits. Instead, we first study the fabrication of masks made by EBID and transfer the masks into the substrate underneath using plasma etching. The pattern transfer process of such small features in itself is quite a challenge. Guan et al. 
demonstrated the pattern transfer into silicon of masks made by EBID using the $\mathrm{W}(\mathrm{CO})_{6}$ or $\mathrm{C}_{10} \mathrm{H}_{8}$ as a precursor, resulting in silicon nanowires with a width of $33 \mathrm{~nm}{ }^{8}$ The etching process, which is based on $\mathrm{SF}_{6}$ and $\mathrm{C}_{4} \mathrm{~F}_{8}$, leads to a selectivity of $\sim 21$. They even succeeded in fabricating nanopillars with sizes down to $13.5 \mathrm{~nm}$, and a selectivity of 18, using a bilayer approach with an EBID mask on top of a polymethylmethacrylate mask. However, the authors do not expect that the bilayer approach has an inherently superior resolution over a single-layer approach. Notargiacomo et al. reported on the pattern transfer of ion and electron deposited Pt masks by using reactive ion etching, where pattern transfer of lines with width down to $100 \mathrm{~nm}$ is obtained with fluorine chemistry. ${ }^{9}$ The same chemistry was used by Heerkens et al., who demonstrated the pattern transfer into silicon of 20-nm-wide lines deposited using EBID from a platinum precursor. They achieved a selectivity of the PtEBID lines to silicon of $\sim 6 .{ }^{10}$ The pattern transfer of EBID masks can be used for many applications, e.g., gold bowtie antennas with $10 \mathrm{~nm}$ gap spacing were fabricated on atomic force microscope (AFM) tips. ${ }^{11}$ Fleischer et al. presented a process for the fabrication of nano-cones by focused electron beam deposition of a local etch mask on a metallized nonplanar surface, and argon ion etching. The smallest resulting cone had a diameter of $60 \mathrm{~nm}$ and height of $55 \mathrm{~nm} .^{12}$ However, as far as we know, the pattern transfer of sub-20 nm single-layer EBID mask structures has not been reported yet.

In this work, we present results of experiments that were carried out to gain experience toward etching with sub-10 nm EBID masks. As sub-10 nm structures deposited by EBID are also very shallow, with a typical aspect ratio of 1 , the pattern transfer into an underlying layer is a method by which the aspect ratio of the structure can be increased. High-resolution EBID patterns were deposited onto a silicon substrate, serving as a mask for the subsequent etching process. Two sets of EBID masks, one with somewhat larger structures between 20 and $40 \mathrm{~nm}$ and one with structures between 8 and $20 \mathrm{~nm}$, were fabricated using the platinum precursor $\mathrm{MeCpPtMe}_{3}$ in an FEI Nova Nano 650 Dual Beam system. The masks consist of arrays of dots and sets of lines, with sizes in the range between 40 and $8 \mathrm{~nm}$. We used different etching processes, based on hydrogen bromide, chlorine, and fluorine chemistries, which are known to etch silicon.

\section{Experiments}

Basically two sets of experiments were performed. The first experiments were carried out to get some experience with pattern transfer into silicon using EBID structures as etching mask. Larger structures are preferred, because they are easier to find after the etching process. The second set of experiments is then carried out to demonstrate the pattern transfer into silicon of sub-10 nm EBID masks, with deposits of size down to $8 \mathrm{~nm}$.

The quality of the etching process is mostly determined by the directionality of the etching. Preferably, the etch is anisotropic, very selective, and results in smooth surfaces after etching. Determining the directionality of the etching is challenging as the resulting structures are really small. The change in width of the structures gives an indication of the directionality.

Inspection of the masks, before and after etching, is done both in an FEI Nova Nano Lab 650 SEM and in a Bruker
Nanoscope V AFM. The lateral dimension of the structures is manually measured in the SEM images, while the height is calculated from the AFM profile.

The smoothness of the resulting surface after etching is an important factor with the goal in mind to fabricate single nanometer devices. To characterize the etching process, usually the etch selectivity is determined, defined as the ratio of the etching rate of the substrate material to the etching rate of the mask material. Because of the dimensions of the structures, it is difficult to measure the selectivity of the tested etching processes. Hence, we chose to characterize the process by the height ratio, defined as the ratio between the height of the mask before etching and the height of the structure after etching. This parameter gives a hint to what the etching rates of the materials are. A large height ratio is preferred because it leads to higher aspect ratio structures.

The first set of 20- to 40-nm EBID masks was fabricated using the platinum precursor $\mathrm{MeCpPtMe}_{3}$ in an FEI Nova Nano Lab 650 SEM, using a $30 \mathrm{kV}$ beam focused in a 40 pA spot, in high-resolution mode. We used silicon substrates partially covered by $60 \mathrm{~nm}$ of molybdenum. Because the total size of the EBID mask is only $4 \mu \mathrm{m}$, a search pattern is necessary to be able to retrieve the structures after pattern transfer. Hence, an array of $13 \times 13$ circular holes of $\sim 10 \mu \mathrm{m}$ diameter has been lithographically opened in the molybdenum layer using photoresist as a mask, leading to a visible structure that can be easily retrieved. Figure 1 shows the process flow for the sample fabrication. The sacrificial layer (photoresist) is deposited on top of the silicon substrate. By means of optical lithography, the array of circles is transferred into the photoresist layer. A molybdenum layer is deposited on the sample surface after which the resist is removed in a lift-off process. The EBID structures are then deposited inside one of these circles, directly on the silicon surface.

The EBID masks, patterned with the Pt precursor, typically consist of carbon $(85 \%)$ and $\mathrm{Pt}(15 \%)$. The first set of masks consists of four arrays of 5 by 5 dots, with a size in the sub-40 nm range. Figure 2 shows the SEM micrograph and the AFM image of one of the patterned EBID masks, whereas Table 1 shows the patterning parameters for each array of dots. Each dot is defined as a square, the size of which is determined by the number of pixels exposed by the electron beam and the pixel-to-pixel distance. The latter was chosen equal to the diameter of the electron beam, typically $<2 \mathrm{~nm}$. The beam is scanned from one spot (pixel) to the next one following a serpentine writing strategy. The beam resides at each pixel for a certain amount of time, called the dwell time. The whole pattern is written 1000 times (the number of passes). We will refer to array A as the top left array of dots, B the top right, $\mathrm{C}$ the bottom left, and D the bottom right. The diameter of the dots varies from 40 to $19 \mathrm{~nm}$, while the height of the dots in array $\mathrm{A}$ is $\sim 4.7 \mathrm{~nm}$.

Because the mask is shallow, it is important that the chosen chemistry is very selective toward the EBID structures. The etching process used to transfer the EBID structures into the underlying silicon is carried out by inductively coupled plasma (ICP) etching, performed in a PlasmaLab System 133 ICP380 at the Oxford Instruments Plasma Technology facility in the United Kingdom. Plasma-based etching allows processes to be carried out at relatively low temperature by lowering the activation energy of the 


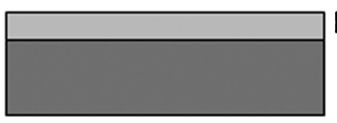

Photoresist

Silicon

substrate

2)

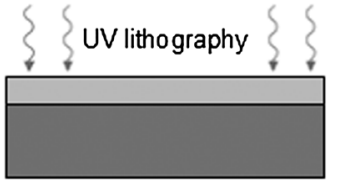

Photoresist

Silicon

substrate

3)

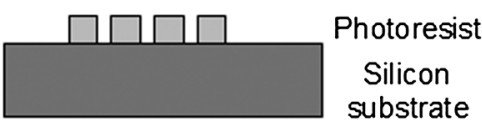

4)

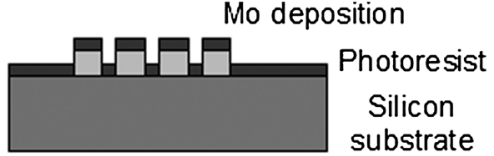

5)

PR removal
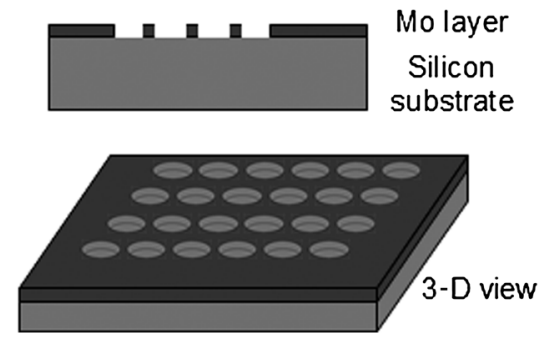

Fig. 1 Process flow for the fabrication and three-dimensional (3-D) view of the search pattern on the Si sample.

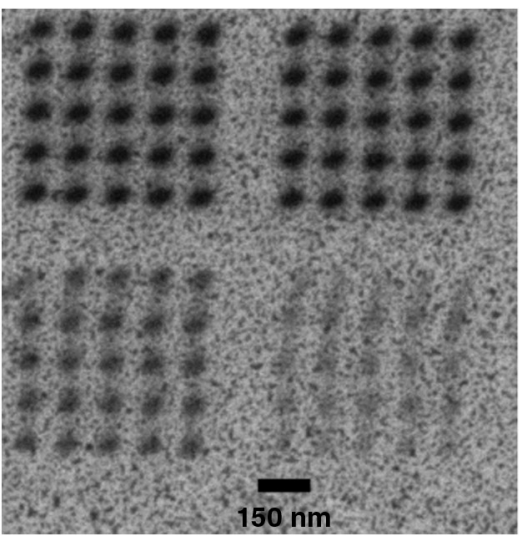

(a)

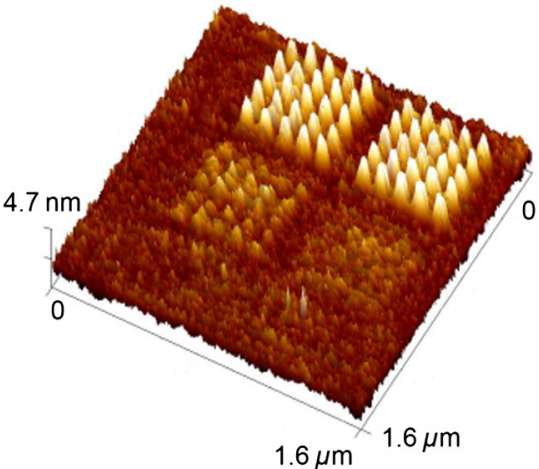

(b)

Fig. 2 (a) Scanning electron microscope (SEM) micrograph of the 20- to 40-nm electron beam-induced deposition (EBID) masks and (b) atomic force microscope (AFM) 3-D view of the 20- to 40-nm EBID mask.

chemical etching reactions. Moreover, plasma etching allows a wide area to be processed, providing uniform etching and control over the direction of etching. Figure 3 shows the schematic of the ICP etching tool. By applying a combination of RIE power at the lower electrode and ICP power to the coils around the upper chamber, a better control over the ion density and the ion energy is provided. The wafer is mechanically clamped to the fluid-cooled lower electrode

Table 1 Electron beam-induced deposition (EBID) parameters for the 20- to $40-\mathrm{nm}$ masks.

\begin{tabular}{lccc}
\hline Array & $\begin{array}{c}\text { Size of each } \\
\text { dot (pixel) }\end{array}$ & $\begin{array}{c}\text { Dwell } \\
\text { time }\end{array}$ & $\begin{array}{c}\text { Number of } \\
\text { passes }\end{array}$ \\
\hline A (top left) & $15 \times 15$ & $400 \mathrm{~ns}$ & 1000 \\
B (top right) & $10 \times 10$ & $800 \mathrm{~ns}$ & 1000 \\
C (bottom left) & $5 \times 5$ & $1 \mu \mathrm{s}$ & 1000 \\
D (bottom right) & $5 \times 5$ & $400 \mathrm{~ns}$ & 1000 \\
\hline \hline
\end{tabular}

and helium is used as the sample backside cooling gas for better control over the wafer temperature. The backside cooling gas mixes with the etching gas in the chamber, but it has almost no impact on the process, being light, hard to ionize, and chemically inactive. The sample pieces were bonded to a carrier wafer to ensure good temperature control. A gas inlet leads the gas mixture into the chamber, which is at a controlled pressure and temperature. ${ }^{13}$

These masks are etched using hydrogen bromide $(\mathrm{HBr})$, chlorine $\left(\mathrm{Cl}_{2}\right)$, and boron trichloride $\left(\mathrm{BCl}_{3} / \mathrm{Cl}_{2}\right)$ chemistries, which are known to selectively etch silicon when using carbon-based masks. These etching processes are carried out by using both ICP and RIE power. $\mathrm{HBr}$ chemically reacts with silicon atoms on the surface, forming $\mathrm{Si}_{x} \mathrm{Br}$, a compound with low volatility. ${ }^{13}$ This characteristic renders $\mathrm{HBr}$ a highly anisotropic silicon etchant, in which $\mathrm{Si}_{x} \mathrm{Br}$ forms a sidewall protective layer. Chlorine is also known to etch silicon either in a pure form or as part of the gas mixture $\mathrm{BCl}_{3} / \mathrm{Cl}_{2}$. ${ }^{14}$ When using either pure $\mathrm{Cl}_{2}$ or $\mathrm{BCl}_{3} / \mathrm{Cl}_{2}$ chemistry, silicon atoms bind to $\mathrm{Cl}_{2}$ forming the volatile compound $\mathrm{SiCl}_{x}$, which can be pumped out of the system. Chlorine etching is strongly dependent on crystallographic orientation, leading to an anisotropic etch profile. ${ }^{15}$ Once the properties of 


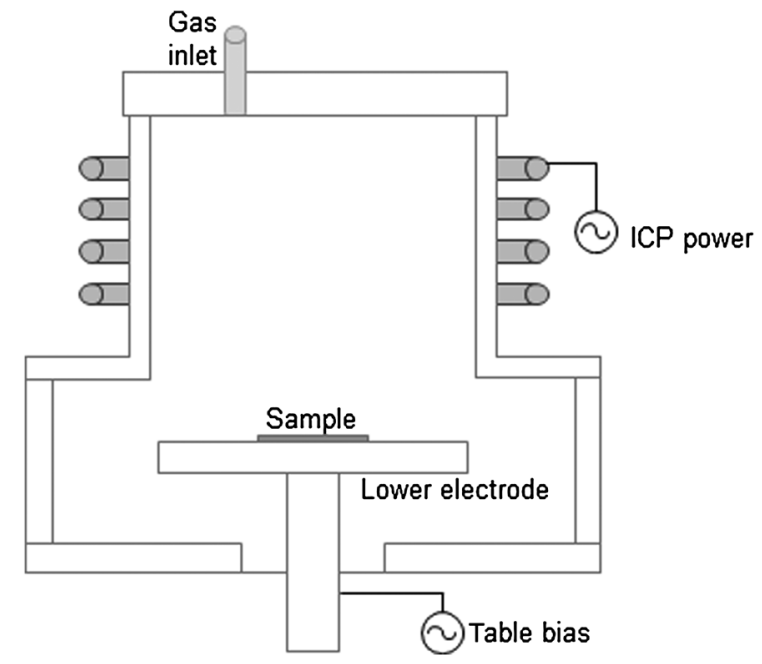

Fig. 3 Inductively coupled plasma reactive ion etch plasma etching tool used in the Oxford Instruments Plasma Technology facility.

each chemistry are determined, they can be further tested on even smaller EBID masks, to demonstrate the pattern transfer of sub-10 nm structures.

For that purpose, the second set of 8- to 20-nm EBID masks was fabricated in the same setup as the first set of masks, with a $20 \mathrm{kV}$ beam, focused in a $40 \mathrm{pA}$ spot, but now in ultrahigh-resolution mode. In this case, we used a different substrate than the molybdenum-silicon one. The reason for this is that the smoothness of the molybdenum layer makes focusing of the electron beam a difficult process. We decided to use the same search pattern, but instead of depositing a metal layer outside the array of circular holes, we plasma-etched the silicon for a very short time using $\mathrm{SF}_{6}$ and oxygen such that black silicon is formed at $\sim 20 \mathrm{~nm}$ below the unetched silicon surface. Figure 4 shows the process flow of the fabrication of the black silicon samples used for the fabrication of the 8- to 20-nm EBID masks.

In this configuration, the EBID patterns are deposited inside the circles on top of unetched silicon, surrounded by the pillar-shaped black silicon, on which focusing the beam is very easy. Figure 5(a) shows an 8- to 20-nm EBID mask. It consists of three arrays of 5 by 5 dots and nine sets of lines. The dots have sizes of $\sim 20,13$, and $7 \mathrm{~nm}$ and a height of 7 ,

1)

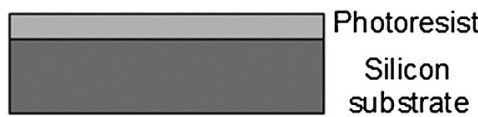

2)

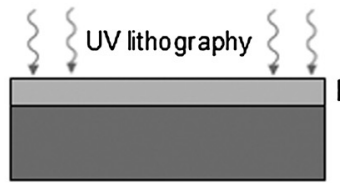

Photoresist Silicon substrate

3)

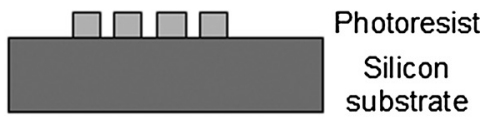

2.5 , and $1 \mathrm{~nm}$, for the top left, central, and right array, respectively. Each set of lines consists of 10 lines with 100, 50, and $20 \mathrm{~nm}$ spacing. The width of these lines is 17,10 , and $8 \mathrm{~nm}$, while the height is 5,2, and $0.8 \mathrm{~nm}$ for the first, second, and third column, respectively. Figure 5(b) shows the high-resolution image of the $8 \mathrm{~nm}$ set of lines, with 50-nm spacing.

Table 2 shows the deposition parameters and the designed pixel size of each structure of the mask. In this case, a singlepass patterning mode is chosen, instead of a multipass strategy. The deposition occurs in the current limited regime in which there is an abundant supply of precursor molecules. Hence, the resulting deposits will not be affected by the chosen writing strategy (single pass or multipass), as demonstrated by Hari et al. ${ }^{16}$ The multipass strategy is preferred because it is less sensitive to drift.

The sample fabrication and subsequent inspection, which must be done before and after the etching, is a very time-consuming process; hence, the amount of samples available is limited. Therefore, the etching chemistries for the 8- to 20-nm masks have to be chosen carefully. The chemistries chosen are $\mathrm{HBr}, \mathrm{Cl}_{2}$, and fluorine $\left(\mathrm{SF}_{6} / \mathrm{C}_{4} \mathrm{~F}_{8}\right)$, for reasons that will be discussed. Chemical etching by fluorine is known to be isotropic; therefore, it is necessary to add a component that provides anisotropic etching. We used an $\mathrm{SF}_{6}$ plasma, which is known to etch silicon easily, and by adding $\mathrm{C}_{4} \mathrm{~F}_{8}$, a fluorocarbon film is deposited that protects the sidewalls of the etched structure and sustains the anisotropy of the etching process. However, the ratio between carbon and fluorine must be tuned carefully, because polymer deposition can occur when the F-to-C ratio is too small.

\section{Results and Discussion}

Initial experiments were carried out on 20- to 40-nm etching masks to demonstrate the possibility of using EBID masks for nano-patterning into underlying silicon. In this case, $\mathrm{HBr}$, $\mathrm{Cl}_{2}$, and $\mathrm{BCl}_{3} / \mathrm{Cl}_{2}$ chemistry were chosen because of their affinity to etch silicon better than carbon, i.e., the main element in the EBID deposit when using the Pt precursor. In case of $\mathrm{HBr}$, helium, which is used as a sample backside cooling gas, is also pumped into the system. A combination of ICP and RIE power is used (750 and $100 \mathrm{~W}$, respectively), at a pressure of $10 \mathrm{mTorr}$ and a temperature of $30^{\circ} \mathrm{C}$, for 1 min. Figure 6(a) shows an SEM micrograph of the etched mask: as the EBID masks were completely etched, the dots

4)

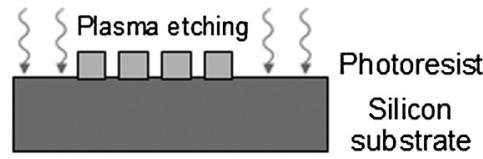

5)

PR removal
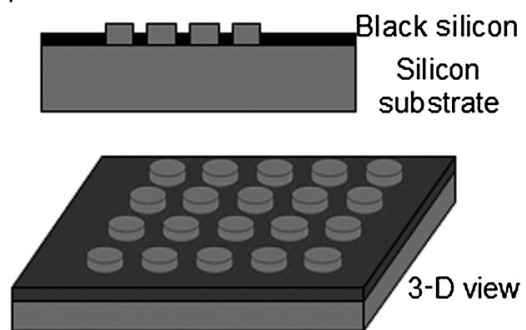

Fig. 4 Process flow for the fabrication and 3-D view of the optimized search pattern on the Si sample. 


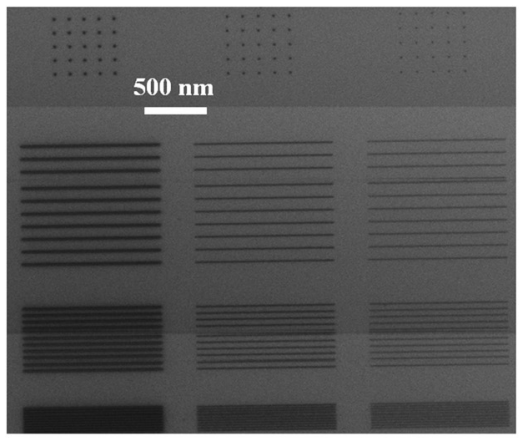

(a)

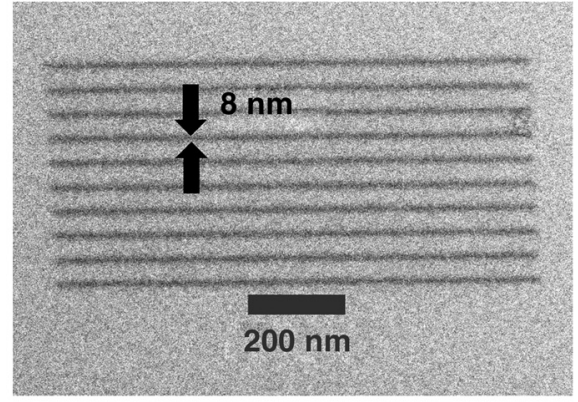

(b)

Fig. 5 (a) SEM micrograph of the sub-20 nm EBID mask, and (b) high-resolution SEM micrograph of the $8-\mathrm{nm}$ lines at $50 \mathrm{~nm}$ spacing.

Table 2 EBID parameters for the 8- to $20-\mathrm{nm}$ masks.

\begin{tabular}{lccc} 
Array & Size (pixel) & Dwell time & Number of passes \\
\hline D-A (top left) & $12 \times 12$ & $100 \mu \mathrm{s}$ & 1 \\
D-B (top center) & $8 \times 8$ & $100 \mu \mathrm{s}$ & 1 \\
D-C (top right) & $5 \times 5$ & $100 \mu \mathrm{s}$ & 1 \\
\hline Set of lines & Size (pixel) & Dwell time & Number of passes \\
\hline L-A (left) & $1000 \times 1$ & $1 \mathrm{~ms}$ & 1 \\
L-B (center) & $1000 \times 2$ & $100 \mu \mathrm{s}$ & 1 \\
L-C (right) & $1000 \times 1$ & $100 \mu \mathrm{s}$ & 1 \\
\hline \hline
\end{tabular}

consist of silicon only, leading to a poor contrast in the secondary electron image. Figure 6(b) shows the same mask as in Fig. 6(a) compared to the AFM three-dimensional view, where the dots are more visible. The AFM height profile of the central horizontal row of dots, before and after etching, is shown in Fig. 6(c). We observe that this chemistry leads to a rough surface and provides a height ratio (after etching: before etching) of $<2$. The diameter of the dots remained constant, as observed from the SEM images. In this case, only the largest EBID dots could be retrieved after etching.

By inserting a dummy silicon sample in the plasma chamber, next to that with the EBID masks, it is possible to measure the etch rate of silicon. The same can be done in case of silicon oxide. The etch rate of the silicon oxide is measured to be $\sim 2 \mathrm{~nm} / \mathrm{min}$, whereas that of silicon is $\sim 46 \mathrm{~nm} / \mathrm{min}$. We conclude that the etch rate of the native silicon oxide is comparable or maybe even higher than that of the EBID

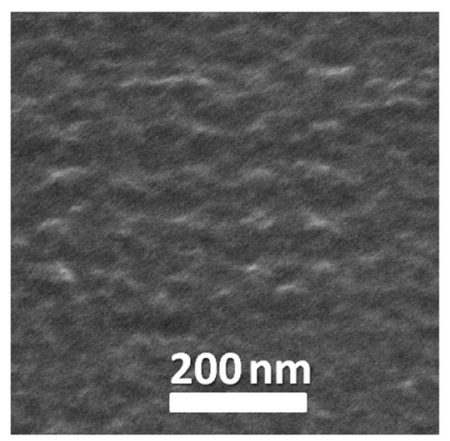

(a)

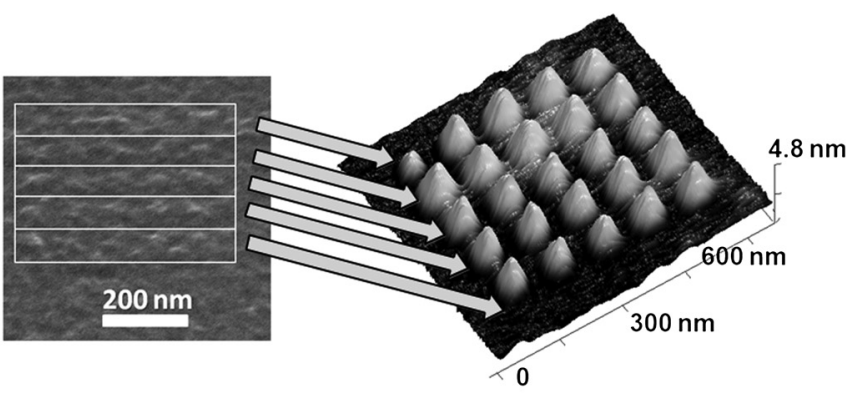

(b)

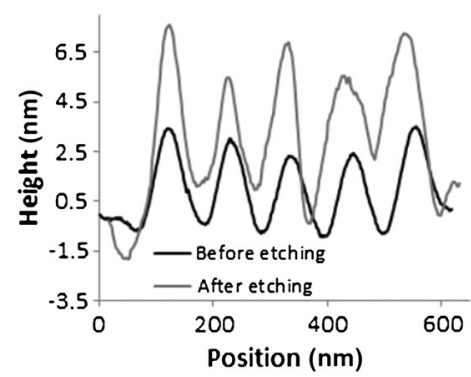

(c)

Fig. 6 (a) SEM micrograph of the array of dots A after etching (HBr chemistry), (b) SEM micrograph and AFM 3-D profile of the same array of dots, and (c) the height profile measured with AFM of the horizontal central row of array $\mathrm{A}$, before and after etching ( $\mathrm{HBr}$ chemistry). 


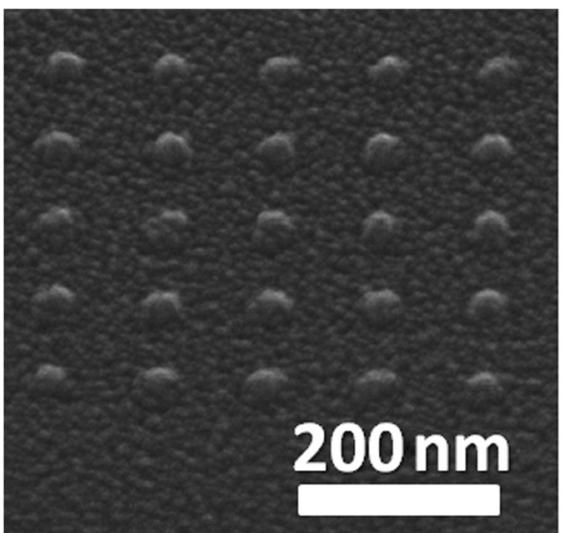

(a)

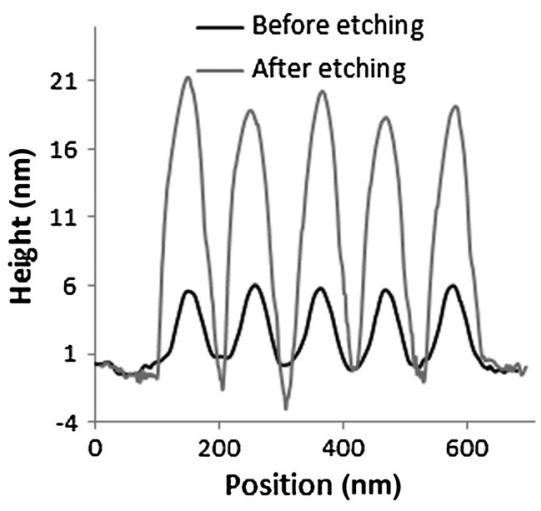

(b)

Fig. 7 (a) SEM micrograph of the array of dots $A$ after etching $\left(\mathrm{Cl}_{2}\right.$ chemistry), and (b) the height profile measured with AFM of the central horizontal row of array $A$, before and after etching $\left(\mathrm{Cl}_{2}\right.$ chemistry).

deposits, causing complete etching of the mask before reaching the silicon substrate.

In case of pure chlorine chemistry, $50 \mathrm{sccm}$ of $\mathrm{Cl}_{2}$ is led into the chamber, together with backside helium, at 10 mTorr and $30^{\circ} \mathrm{C}$. When etching with $\mathrm{Cl}_{2}$ for $1 \mathrm{~min}$, the surface of the sample became extremely granular, and the EBID dots also. The size of the dots, as estimated from the SEM images [Fig. 7(a)], increased from 40 to $\sim 55 \mathrm{~nm}$. As seen from the AFM profile [Fig. 7(b)], the height ratio is $\sim 3.5$. Although this is an acceptable number, the granularity of the resulting mold will prevent successful fabrication of single-nanometer devices.

Figure 8(a) shows the result after etching with $\mathrm{BCl}_{3} / \mathrm{Cl}_{2}$ chemistry for $1 \mathrm{~min} .50 \mathrm{sccm}$ of $\mathrm{Cl}_{2}$ and $20 \mathrm{sccm}$ of $\mathrm{BCl}_{3}$ are led into the chamber together with helium, at 10 mTorr and $30^{\circ} \mathrm{C}$. In this case, the surface remained smooth, as shown in Fig. 8(a). However, some particles can be seen, close to the etched mask as well as in other region of the samples. The reason for that has not been established yet, but we think this might be caused by dirt in the etching chamber. The height ratio is $\sim 2.5$ [Fig. 8(b)], whereas the diameter, as measured from the SEM image, increased from 40 to $\sim 50 \mathrm{~nm}$.

Analyzing the results, we observe good results in terms of height ratio for the $\mathrm{Cl}_{2}$ etching process. The resulting presence of the granularity must be avoided. The granularity may have been caused by the black spots on the silicon surface observed before etching and visible in Fig. 2. They could be resist residues from the lift-off process and cause the sample surface to become granular after the $\mathrm{Cl}_{2}$ process. This might also explain the roughness of the surface resulting after the $\mathrm{HBr}$ etching process. To prevent this, the substrates for the 8- to 20-nm EBID masks were carefully cleaned in an oxygen plasma before carrying out the etching step. We decided to repeat the hydrogen bromide and the chlorine recipes to verify whether the oxygen plasma cleaning improves the surface roughness. Because of the limited number of samples available, we decided to carry out fluorine chemistry instead of $\mathrm{BCl}_{3} / \mathrm{Cl}_{2}$. Although known to be isotropic, fluorine etches silicon very fast, and by adding other components, such as $\mathrm{C}_{4} \mathrm{~F}_{8}$, a passivation wall can be formed, rendering the etch more anisotropic.

$\mathrm{HBr}, \mathrm{Cl}_{2}$, and $\mathrm{SF}_{6} / \mathrm{C}_{4} \mathrm{~F}_{8}$ chemistries are then tested for the pattern transfer of the 8- to 20-nm EBID masks. In case of the $\mathrm{HBr}$ chemistry, only the biggest set of lines is visible in both the SEM micrograph and the AFM image after the etching is performed. For these lines of $17 \mathrm{~nm}$ width and $5 \mathrm{~nm}$ height, the EBID mask is preserved as it was thicker than the other deposits. In Fig. 9(a), the SEM

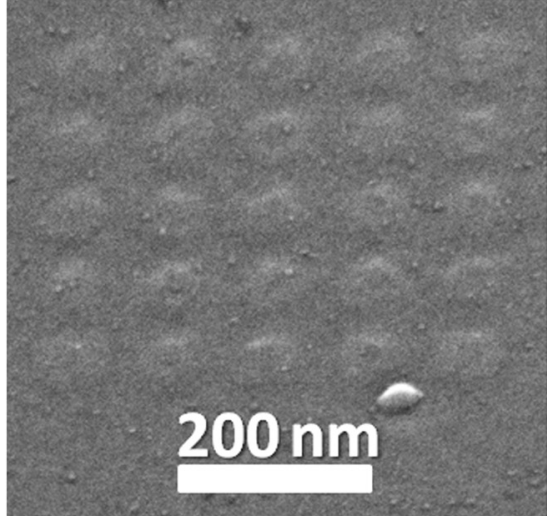

(a)

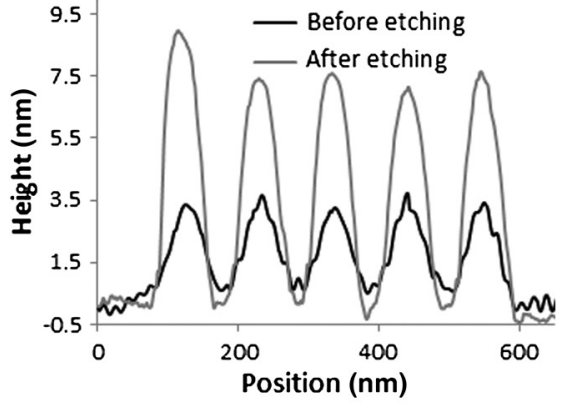

(b)

Fig. 8 (a) SEM micrograph of the array of dots $\mathrm{A}$ after etching $\left(\mathrm{BCl}_{3} / \mathrm{Cl}_{2}\right.$ chemistry), and (b) the height profile measured with AFM of the central horizontal row of array A, before and after etching $\left(\mathrm{BCl}_{3} / \mathrm{Cl}_{2}\right.$ chemistry). 


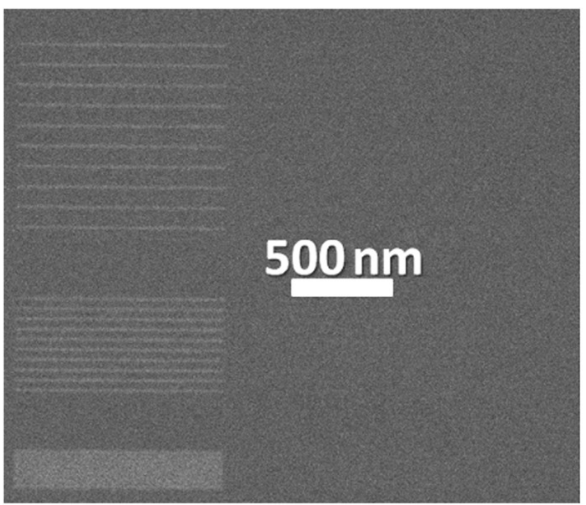

(a)

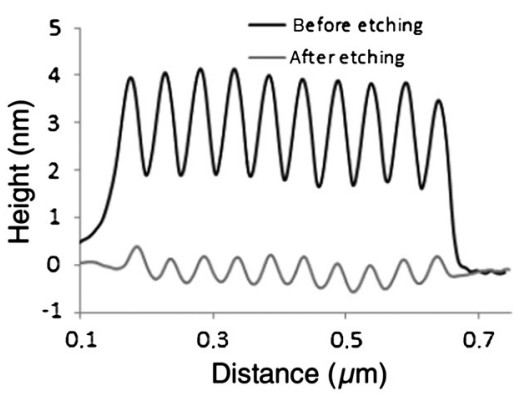

(b)

Fig. 9 (a) SEM micrograph of the EBID mask after etching ( $\mathrm{HBr}$ chemistry), and (b) the integrated height profile measured with AFM of the $17-\mathrm{nm}$ lines with $50 \mathrm{~nm}$ spacing, before and after etching $(\mathrm{HBr}$ chemistry).

micrograph shows the 17 -nm lines with spacing of 100, 50, and $20 \mathrm{~nm}$. In Fig. 9(b), the integrated AFM profile, obtained by integrating over the entire length of the lines, of the 17-nm lines with $50 \mathrm{~nm}$ spacing shows that the height ratio is very low, approximately equal to $1 / 5$. In this case, the etch rate of silicon is measured to be $50 \mathrm{~nm} / \mathrm{min}$ and that of silicon oxide is $1.8 \mathrm{~nm} / \mathrm{min}$. Although the etching rates are comparable to those of the 20- to 40-nm mask experiments, the small height ratio might be caused by a thickening of the native silicon oxide layer during the plasma cleaning. If that is the case, the etch may remove the mask completely before breaking through the oxide layer, because of the slow etching rate of the silicon oxide. Further experiments were carried out to increase the etch rate of the silicon oxide by applying a higher bias voltage; however, no improvements were observed. In case of the $17-\mathrm{nm}$ lines at $50 \mathrm{~nm}$ spacing, the width decreased from 17 to $12 \mathrm{~nm}$ after etching, as estimated from the SEM images. The sample surface, however, is quite smooth and the roughness observed in the case of the 20- to 40-nm mask experiment did not occur. We therefore believe that the roughness caused by the $\mathrm{HBr}$ etching was caused by the photoresist residues on the silicon surface.

In case of pure chlorine etching, when using the same recipe as for the 20- to 40-nm masks, the same granularity is observed, as shown in the SEM image of Fig. 10(a). All sets of lines were visible in both SEM and AFM graphs,

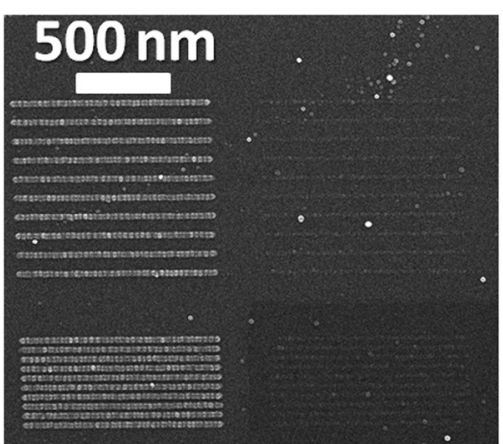

(a)

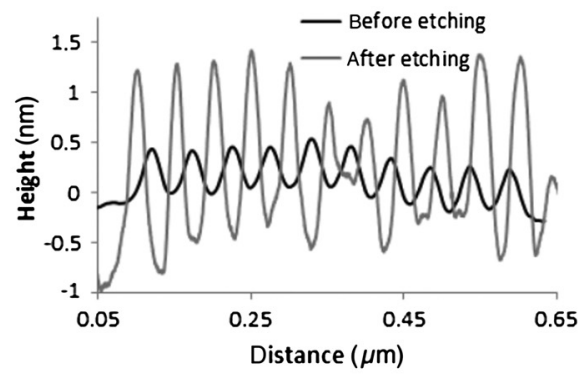

(b)

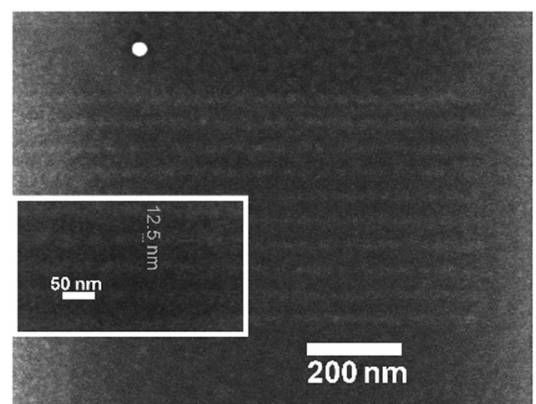

(c)

Fig. 10 (a) SEM micrograph of the EBID mask after etching $\left(\mathrm{Cl}_{2}\right.$ chemistry), (b) the integrated height profile measured with AFM of the $5-\mathrm{nm}$ lines with $50 \mathrm{~nm}$ spacing, before and after etching $\left(\mathrm{Cl}_{2}\right.$ chemistry), and (c) high-resolution SEM micrograph showing the widening of the 8-nm lines to $12.5 \mathrm{~nm}$. 


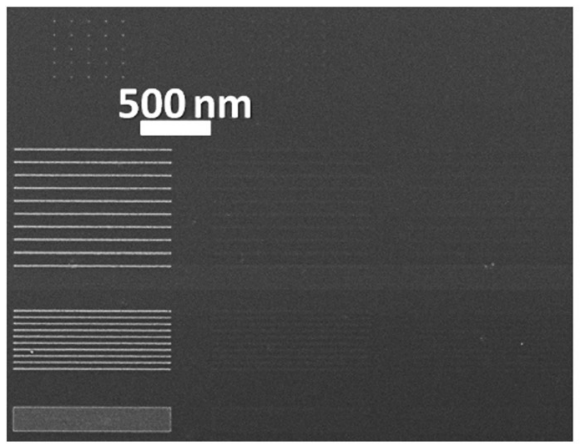

(a)

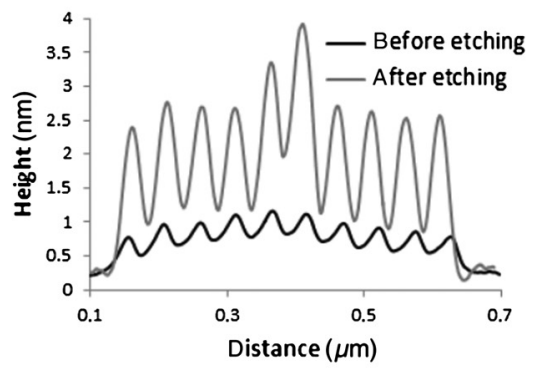

(b)

Fig. 11 (a) SEM micrograph of the EBID mask after etching (fluorine chemistry), and (b) the integrated height profile measured with AFM of the 8-nm lines with $50 \mathrm{~nm}$ spacing, before and after etching (fluorine chemistry).
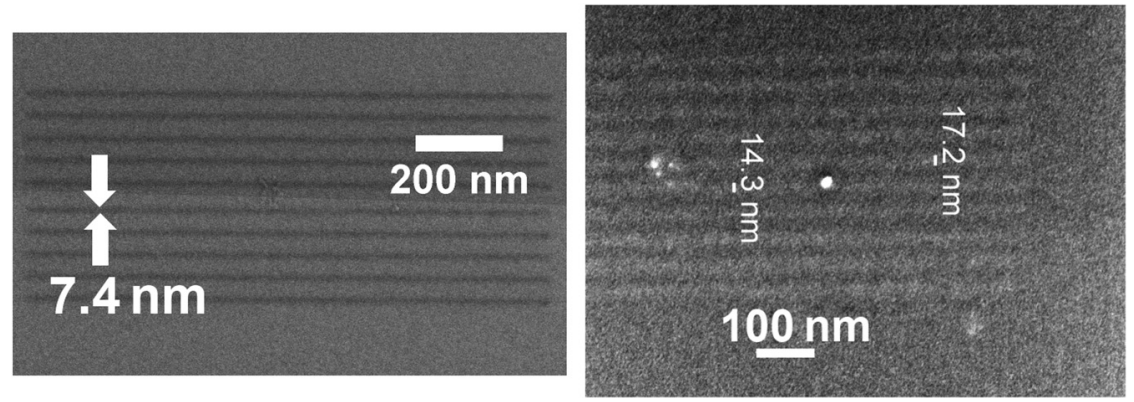

Fig. 12 (a) SEM micrograph of the EBID mask, zoom-in of the region of the 8-nm lines at $50 \mathrm{~nm}$ spacing before etching (fluorine chemistry), and (b) SEM micrograph of the EBID mask; a zoom-in of the region of the 8-nm lines at $100 \mathrm{~nm}$ spacing, after etching (fluorine chemistry).

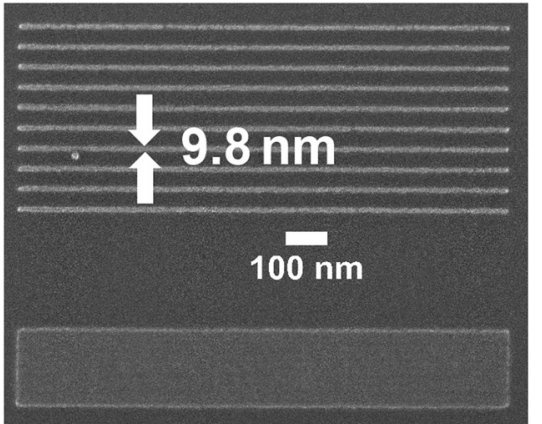

(a)

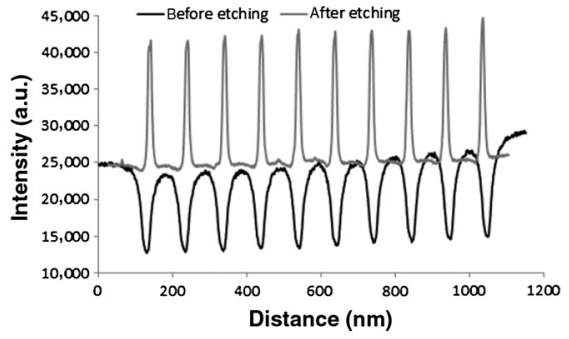

(b)

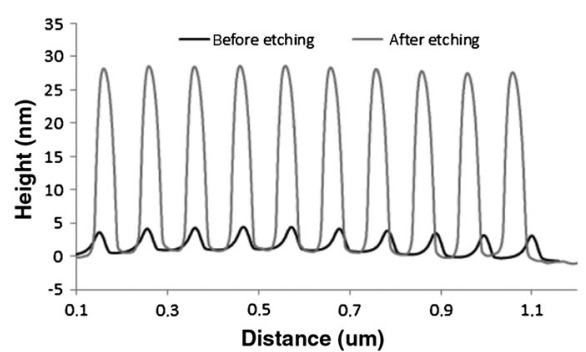

(c)

Fig. 13 (a) SEM micrograph of the EBID mask, zoom-in of the region of the 17-nm lines at $50 \mathrm{~nm}$ spacing, after etching (fluorine chemistry), (b) integrated secondary electron intensity of the 17-nm lines at $50 \mathrm{~nm}$ spacing, before and after etching (fluorine chemistry), and (c) AFM integrated profile of the 17-nm lines at $50 \mathrm{~nm}$ spacing, before and after etching (fluorine chemistry). 
and the integrated AFM profile along the 8-nm lines at $50 \mathrm{~nm}$ spacing, depicted in Fig. 10(b), shows a height ratio of $\sim 3.5$. For these lines, the width increased from 8 to $12.5 \mathrm{~nm}$. Note that some particles close to the EBID deposit are seen in Fig. 9(a), but they were also found in regions of the sample that have not been exposed to the electron beam while fabricating the etching mask. Their origin has not been established yet; however, we believe it can be caused by dirt in the etching chamber.

Last, fluorine etching chemistry is tested on an 8- to 20$\mathrm{nm}$ mask. In this test, $25 \mathrm{sccm}$ of $\mathrm{SF}_{6}$ is mixed together with $50 \mathrm{sccm}$ of $\mathrm{C}_{4} \mathrm{~F}_{8}$, at a pressure of $15 \mathrm{mTorr}$ and a controlled temperature of $15^{\circ} \mathrm{C}$, and at $1200 \mathrm{~W}$ ICP and $30 \mathrm{~W}$ RIE. The etch rate of silicon in this condition is measured to be $\sim 160 \mathrm{~nm} / \mathrm{min}$, whereas that of the native oxide is $20 \mathrm{~nm} /$ min. The resulting lines appear to be smooth in the SEM micrograph, as depicted in Fig. 11(a). No granularity or roughness is encountered and the height ratio calculated from the integrated AFM line profile in Fig. 10(b) is $\sim 5$. The graph in Fig. 11(b) depicts the AFM integrated profile of the 8-nm lines at $50 \mathrm{~nm}$ spacing. The bump located in the fourth and fifth lines corresponds to the presence of a particle accidentally deposited on top of these lines. For these lines, the width increased from 7.4 to $14.3 \mathrm{~nm}$ approximately, as shown in Fig. 12. The contrast of the SEM micrograph in Fig. 12(b) is extremely poor as the EBID mask has been completely removed during the etching process. The secondary electron image therefore only shows topographical contrast and no material contrast. This renders the measurement of the line width rather difficult.

The sets of 17-nm lines emit more secondary electrons than the others, as shown in Fig. 11(a), meaning the EBID mask was sufficiently thick to remain. Figure 13(a) shows the high-resolution SEM micrograph of the 17-nm lines at $50 \mathrm{~nm}$ spacing after etching. The width of the lines is measured to be $9.8 \mathrm{~nm}$, suggesting that the line width decreased. This could also be concluded from Fig. 13(b), which shows the integrated line profile of the secondary electron intensity emitted by the $17 \mathrm{~nm}$ set of lines with $50 \mathrm{~nm}$ spacing before and after etching. However, the AFM profile in Fig. 13(c) reveals a broadening of the lines after etching, and a height ratio of $\sim 8$. This broadening, however, could be partly caused by the finite size of the AFM tip. Our conclusion is that is the criterion for line width determination from secondary electron images is not obvious.

\section{Summary and Conclusions}

EBID is a direct write lithography technique, which can be used to pattern high-resolution structures, with sizes in the sub-10 nm range. Pattern transfer into silicon using masks made by EBID is a method that can potentially be used to fabricate stamps for NIL as it provides an aspect ratio enhancement compared to the aspect ratio of the EBID masks. Initial experiments were carried out using 20- to 40-nm EBID masks, containing dots and lines, to demonstrate the possibility of using EBID masks for nano-patterning into underlying silicon. In this case, etching chemistries based on $\mathrm{HBr}, \mathrm{Cl}_{2}$, and $\mathrm{BCl}_{3}$ were chosen for their affinity in anisotropically etching silicon when using carbon-based masks. When etching with $\mathrm{HBr}$ chemistry, the EBID masks are transferred into underlying silicon with a height ratio, i.e., the ratio of the height of the structures before etching and after etching, of $\sim 2$, whereas the diameter of the dots remained constant. The roughness of the surface observed after the $\mathrm{HBr}$ etching might be caused by some residuals of photoresist, used for the fabrication of the samples. Chlorine etching leads to a height ratio of $\sim 3.5$ and the size of the dots increased from 40 to $55 \mathrm{~nm}$. However, in this case, we observed a surface granularity, which was shown not to be caused by the photoresist residuals. $\mathrm{BCl}_{3}$ mixed with pure chlorine provides a height ratio of $\sim 2.5$, while the size of the dots increased from 40 to $50 \mathrm{~nm}$. When using this chemistry, no surface roughening occurs. Further experiments were carried out with even smaller EBID masks to demonstrate the pattern transfer of sub-10 nm structures. For this purpose, EBID masks consisting of lines with sizes varying from 17 to $8 \mathrm{~nm}$ were fabricated. In order to avoid the roughening and the granularity of the etched samples, the EBID masks were further cleaned in an oxygen plasma. This indeed led to smooth surfaces after $\mathrm{HBr}$ chemistry. The height ratio is now smaller than $1 / 5$, probably because of the thickening of the native oxide layer as a result of the plasma cleaning. Because the etch rate of the silicon oxide using the hydrogen bromide chemistry is low compared to that of the EBID deposits, it is not possible to break through the oxide layer without removing the mask. In case of pure chlorine etching, the height ratio remained $\sim 3.5$, as in the case of the 20 - to $40-\mathrm{nm}$ masks, and the lines widened from 8 to $12.5 \mathrm{~nm}$. However, the granularity can still be observed on the sample surface as well as on the lines themselves, meaning that this is an inherent effect of the $\mathrm{Cl}_{2}$ etch. Very good results are obtained with the fluorinebased etching, in terms of height ratio and surface smoothness. In case of the 8-nm lines, the height ratio is $\sim 5$ and the width increased from 7.4 to $14.3 \mathrm{~nm}$. However, because the mask was completely removed during the etching, the lines are made of only silicon, causing a very poor contrast in the secondary electron image. The 17-nm EBID lines, being also thicker than the 8-nm lines, were not completely etched away and the resulting lines have a width that is measured to be $9.8 \mathrm{~nm}$. In summary, we have demonstrated that the pattern transfer of 7.4-nm-wide lines into silicon, using chlorine and fluorine chemistries, resulted in slightly broader lines of $14.3 \mathrm{~nm}$ width.

\section{Acknowledgments}

The research leading to these results has received funding from the European Union's Seventh Framework Programme FP7/2007-2013 under Grant Agreement No. 318804 (SNM: Single Nanometer Manufacturing for beyond CMOS devices).

\section{References}

1. W. F. van Dorp et al., "Approaching the resolution limit of nanometerscale electron beam-induced deposition," Nano Lett. 5(7), 1303-1307 (2005).

2. J. C. van Oven et al., "Electron-beam-induced deposition of 3-nm-halfpitch patterns on bulk Si," J. Vac. Sci. Technol. B 29, 06F305 (2011).

3. P. C. Post et al., "Parallel electron-beam-induced deposition using a multi-beam scanning electron microscope," J. Vac. Sci. Technol. B 29, 06F310 (2011).

4. A. Mohammadi-Gheidari, C. W. Hagen, and P. Kruit, "Multibeam scanning electron microscope: experimental results," J. Vac. Sci. Technol. B 28, C6G5 (2010).

5. A. Mohammadi-Gheidari and P. Kruit, "Electron optics of multi-beam scanning electron microscope," Nucl. Instrum. Methods Phys. Res. A 645, 60-67 (2011). 
6. L. J. Guo, "Nanoimprint lithography: methods and material requirements," Adv. Mater. 19, 495-513 (2007).

7. D. Morecroft et al., "Sub-15 nm nanoimprint molds and pattern transfer," J. Vac. Sci. Technol. B 27, 2837-2840 (2009).

8. Y. Guan et al., "Nanoscale lithography via electron beam induced deposition," Nanotechnology 19, 505302 (2008).

9. A. Notargiacomo, E. Giovine, and L. Di Gaspare, "Ion and electron beam deposited masks for pattern transfer by reactive ion etching," Microelectron. Eng. 88, 2710-2713 (2011).

10. C. Th. H. Heerkens et al., "Electron beam induced deposited etch masks," Microelectron. Eng. 86, 961-964 (2009).

11. A. Weber-Bargioni et al., "Functional plasmonic antenna scanning probes fabricated by induced-deposition mask lithography," Nanotechnology 21, 065306 (2010).

12. M. Fleisher et al., "Fabrication of metallic nanocones by induced deposition of etch masks and ion milling," Microelectron. Eng. 88, 22472250 (2011).

13. A. L. Goodyear et al., "High resolution inductively coupled plasma etching of $30 \mathrm{~nm}$ lines and spaces in tungsten and silicon," $J$. Vac. Sci. Technol. B 18, 3471-3475 (2000).

14. A. Kassam et al., "Characterization of $\mathrm{BCl} 3-\mathrm{Cl} 2$ silicon trench etching," J. Electrochem. Soc. 137(5), 1613-1617 (1990).

15. D. L. Flamm, "Mechanisms of silicon etching in fluorine- and chlorinecontaining plasmas," Pure Appl. Chem. 62(9), 1709-1720 (2009) http://dx.doi.org/10.1351/pac199062091709

16. S. Hari et al., "Size and shape control of sub- $20 \mathrm{~nm}$ patterns fabricated using focused electron beam-induced processing," J. Micro/Nanolith MEMS MOEMS 13, 033002 (2014)

Marijke Scotuzzi is a PhD student at Delft University of Technology. She received her BSc degree in information engineering in 2010 and her MSc degree in electronic engineering from the University of Brescia, Italy, in 2013. She joined Delft University of Technology in 2013, and her current research interests include electron optics, nanofabrication using electron beam induced deposition, and electron dispersive x-ray analysis of nanostructures.

Martin J. Kamerbeek received his MSc degree in 2008 in electrical engineering from the Delft University of Technology. He currently designs micro- and nanofabrication processes at Mapper Lithography and at the Delft University of Technology in the Charged Particle Optics group.

Andy Goodyear is principal applications specialist with Oxford Instruments Plasma Technology, where he has worked since 1995 developing equipment and processes for advanced plasma etching and deposition applications. He was previously employed at Philips Research Laboratories, Redhill (1985 to 1995), developing PECVD, plasma etch, and e-beam lithography processes for a variety of applications.

Mike Cooke is chief technology officer for Oxford Instruments Plasma Technology, where he has worked since 1992. He is interested in developing techniques for etching and depositing thin films using low-pressure plasma and ion beams.

Cornelis W. Hagen received his MSc degree in 1983 and his PhD degree in 1991 from the Free University of Amsterdam, The Netherlands. He was a researcher at the Paul Scherrer Institute in Switzerland from 1989 to 1992 and at the Kamerlingh Onnes Laboratory of Leiden University, The Netherlands, from 1992 to 1994. In 1994, he joined Delft University of Technology as an assistant professor and was appointed as an associate professor in 2008. His area of research is microscopy and lithography with charged particles. 